\title{
The coexistence of classical bulges and disky pseudobulges in early-type disk galaxies
}

\author{
Peter Erwin ${ }^{1,2}$ \\ ${ }^{1}$ Max-Planck-Insitut für extraterrestrische Physik, Giessenbachstrasse, \\ 85748 Garching, Germany \\ email: erwin@mpe.mpg.de \\ ${ }^{2}$ Universitäts-Sternwarte München, Scheinerstrasse 1, 81679 München, Germany
}

\begin{abstract}
Close examination of "pseudobulges" in several early-type disk galaxies indicates that they are actually composite structures consisting of both a flattened, kinematically cool disklike structure ("disky pseudobulge") and a rounder, kinematically hot spheroidal structure ("classical bulge"). This indicates that pseudobulges, thought to form from internal secular evolution, and classical bulges, thought to form from rapid mergers, are not exclusive phenomena: some galaxies can have both.
\end{abstract}

Keywords. galaxies: bulges, galaxies: elliptical and lenticular, $\mathrm{cD}$, galaxies: structure, galaxies: individual (NGC 4371)

\section{Introduction}

There has been considerable debate recently on the issue of "pseudobulges": cases where the central "bulge" of a disk galaxy is apparently not a small elliptical galaxy embedded within the disk (the "classical" model of a bulge) but instead something more disklike and - it is thought - the result of internal, secular evolution processes instead of violent early mergers (see the review by Kormendy \& Kennicutt 2004). The tendency has been to contrast pseudobulges and classical bulges as in some sense exclusive categories: galaxies have either a classical bulge (e.g., early-type disks) or a pseudobulge (e.g., late-type disks), but not both at once (but see Athanassoula 2005). Here, I present photometric and kinematic evidence suggesting that some galaxies can have both at once.

\section{Definitions (Or, What I Mean by "Bulge" and "Pseudobulge")}

The term "pseudobulge" has become overly general and confused, with different authors using the term to describe different stellar structures, and using different criteria to identify them. In this study, I recognize and am concerned with three entities:

- Photometric Bulge: This is the region of a galaxy defined by a bulge-disk decomposition, where excess light in the inner regions of a galaxy dominates over the light of the outer exponential disk.

- Classical Bulge: This is the traditional idea of a "bulge": a spheroidal or weakly triaxial collection of stars dominated by random motions (velocity dispersion). It can be recognized by being significantly rounder (in projection) than the disk, and by being kinematically hot.

- (Disky) Pseudobulge: This is an inner disk of stars, with a geometry (flattening) similar to that of the outer disk. A "kinematic" pseudobulge is one with evidence for rotationally dominated stellar kinematics (i.e., more like disk kinematics). Secondary characteristics can include nuclear bars, spirals, rings, etc., but these are not required. 
Note that I do not consider "boxy/peanut-shaped bulges," which are well understood as the vertically thickened inner part of bars. I also do not touch on issues of stellar colors, star formation, or dust; S0 galaxies have been shown to harbor disky pseudobulges even though they are generally free of dust and recent star formation (Erwin, Vega Beltrán, Graham, et al. 2003). Finally, I do not assume that particular surface-brightness profiles (as indicated by, e.g., Sérsic indices) necessarily belong to one category or another, although in practice disky pseudobulges often have quasi-exponential profiles.

\section{Methodology}

The basic approach is to first identify the photometric bulge of a galaxy via a bulgedisk decomposition. The morphology, structure, and geometry of the photometric bulge is then examined, to see if it is predominately disklike or spheroidal. Finally, the stellar kinematics of the photometric bulge are used to determine if the stellar motions are dominated by velocity dispersion (as for a classical bulge) or by rotation.

Kinematically, photometric bulges can be judged by placing them on the well-known $V_{\max } / \sigma$ diagram. Empirically, almost all ellipticals - and a number of unambiguously classical bulges in edge-on spirals - fall on or below the line traced by an idealized isotropic oblate rotator model (IOR; Binney 1978). Following Kormendy (1982), I identify photometric bulges which fall above the IOR curve as kinematic pseudobulges: they are dominated by rotation to a degree not seen in ellipticals and bona fide classical bulges.

All of this follows in the path pioneered by Kormendy (1982). What is new is the idea of a recursive approach once a disky pseudobulge has been identified. By using highresolution imaging (e.g., from HST) and spectroscopy, we can focus on the inner region of the pseudobulge and - in some cases, at least - identify a separate "inner photometric bulge" which has rounder isophotes and hotter kinematics.

\section{Example: NGC 4371}

NGC 4371 is a barred S0 galaxy in the Virgo Cluster, notable for a bright stellar nuclear ring inside its bar (Erwin \& Sparke 1999). In the left-hand panels of Figure 1, I show a major-axis surface-brightness profile, along with a simple bulge-disk decomposition. This decomposition defines the photometric bulge as the region at $r<30^{\prime \prime}$. Inspection of the isophotes (upper middle and upper right panels of Figure 1) shows that this region is precisely where the stellar nuclear ring is found. The ellipticity reaches a peak of $\epsilon=1-b / a=0.40$ at $a \sim 10^{\prime \prime}$; this is close to the ellipticity of the outer disk $(0.45)$.

The stellar kinematics (Figure 1, lower right) show a clear velocity maximum at roughly the same radius as the nuclear ring. The ratio of this velocity to the mean velocity dispersion is $\approx 1.1$, well above the IOR curve given its apparent ellipticity (predicted $V_{\max } / \sigma=0.82$; Figure 2). Since the photometric bulge is both highly flattened (like a disk) and dominated by rotation (kinematically similar to a disk), it qualifies as a pseudobulge. (This was originally pointed out by Kormendy 1982.)

However, close inspection of the pseudobulge using HST images shows that at small radii $\left(r<6^{\prime \prime}\right)$, the isophotes becomes distinctly rounder, with $\epsilon \approx 0.3$. (The minimum in ellipticity just outside this zone is consistent with the isophotal effects of an elliptical ring superimposed on a rounder bulge; see Erwin et al. 2001). A bulge/disk decomposition of the photometric bulge profile shows that it is well fit by an exponential + an inner Sérsic component (along with an asymmetric Gaussian to account for the ring; lower middle panel of Figure 1). The resulting "inner" photometric bulge turns out to be precisely that region $\left(r<6^{\prime \prime}\right)$ where the isophotes become rounder. So it appears that while the outer 

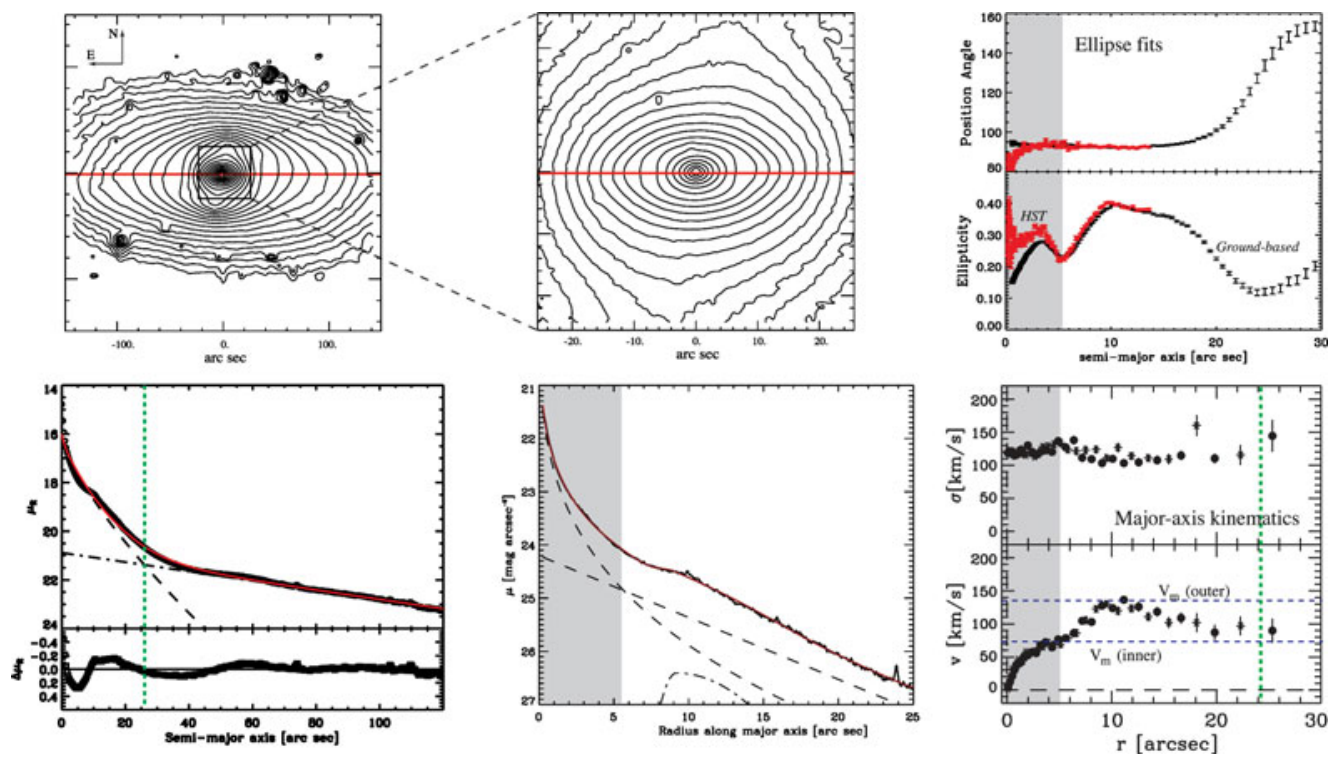

Figure 1. Upper left: $R$-band isophotes of NGC 4371, with major-axis cut line. Lower left: Major-axis surface-brightness profile, with Sérsic + exponential fit. The Sérsic-dominated region (the "photometric bulge") is $r<30^{\prime \prime}$; the vertical dashed green line marks the boundary. Upper middle: zoom of the photometric bulge region, showing elliptical isophotes and rounder isophotes inside. Lower middle: Surface-brightness profile of the photometric bulge region, fit with Sérsic + exponential + ring; an "inner photometric bulge" dominates the light at $r<6$ " (light gray shading). Upper right: Ellipse fits to ground-based and HST isophotes. Lower right: Major-axis stellar kinematics. The stellar velocity has a clear maximum in the photometric bulge region $\left(r \sim 10^{\prime \prime}\right)$, and a plateau in the inner photometric bulge $\left(r \sim 4^{\prime \prime}\right)$.

part of the pseudobulge is highly flattened and nearly exponential in profile, the interior harbors an additional, rounder component. The kinematics in this region (lower right panel, Figure 1) have $V_{\max } / \sigma$ almost identical to the $V_{\max } / \sigma$ predicted for $\epsilon=0.3$. The innermost component thus appears to be a classical bulge: rounder than the pseudobulge (and the outer disk) and not dominated by rotation.

\section{Discussion}

Preliminary analyses have been done for five more galaxies (four S0 and one Sab) which show similar composite structures and kinematics; these are indicated by blue symbols in Figure 2. Note that since these galaxies have bars, they most likely also have the box/peanut structures of bars; these galaxies probably have all three of the distinct components suggested by Athanassoula (2005). Comparison with an ongoing study of pseudobulges in barred S0 galaxies (Erwin, Aguerri, Beckman, et al. 2008) suggests that at least one quarter of S0 pseudobulges may be composite systems.

Two of these composite systems (NGC 2787 and NGC 3384) are known supermassive black hole hosts, which suggests a possible resolution to an issue first raised by Kormendy \& Gebhardt (2001). Black hole masses correlate well with properties of host elliptical galaxies, and also with properties of host (photometric) bulges in disk galaxies, which suggests common evolutionary mechanisms. However, black holes are apparently also found in galaxies with pseudobulges, which are thought to have formation mechanisms different from those of ellipticals and classical bulges. If pseudobulge galaxies with black 


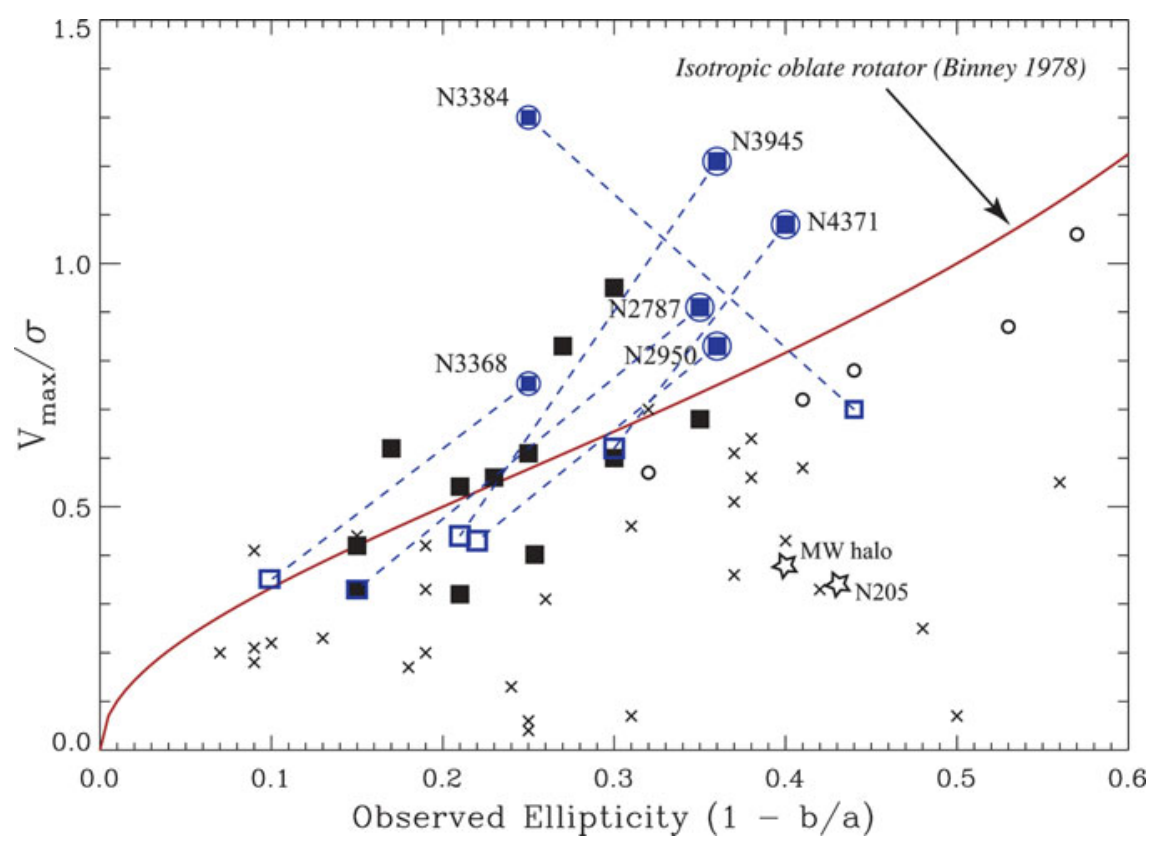

Figure 2. $V_{\max } / \sigma$ diagram. Crosses are elliptical galaxies (Davies et al. 1983), open circles are large, edge-on bulges (Kormendy \& Illingworth 1982; Jarvis \& Freeman 1985), and stars are dE galaxy NGC 205 (Geha et al. 2006) and the Milky Way halo (Ibata et al. 2007 and references therein). Filled black squares are photometric bulges of barred S0 galaxies (Erwin et al. 2008); circled/filled blue squares are disky pseudobulges with inner classical bulges (open blue squares), with dashed lines connecting classical bulges and disky pseudobulges of the same galaxy.

holes also have classical bulges, then the idea of a common evolutionary mechanism driving black hole and (classical) bulge growth may still be tenable.

\section{References}

Athanassoula, E. 2005, MNRAS 358, 1477

Binney, J. 1978, MNRAS 183, 501

Davies, R. L., Efstathiou, G., Fall, S. M., Illingworth, G., \& Schechter, P. L. 1983, ApJ 266, 41 Erwin, P. \& Sparke, L. S. 1999, ApJ (Letters) 521, L37

Erwin, P., Vega Beltrán, J. C., \& Beckman, J. E. 2001, in: J.H. Knapen, J.E. Beckman, I. Shlosman \& T.J. Mahoney (eds.), The central kpc of starbursts and AGN: the La Palma connection (San Francisco: ASP Conference Series), 171

Erwin, P., Vega Beltrán, J. C., Graham, A. W., \& Beckman, J. E. 2003, ApJ 597, 929

Erwin, P., Aguerri, A., Beckman, J. E., García, T., \& Balcells, M. 2008, in prep

Geha, M., Guhathakurta, P., Rich, R. M., \& Cooper, M. C. 2006, AJ 131, 332

Ibata, R., Martin, N. F., Chapman, S., Ferguson, A. M. N., Lewis, G. F., \& McConnachie, A. W. 2007, ApJ, submitted (arXiv:0704.1318)

Jarvis, B. J. \& Freeman, K. C. 1985, ApJ 295, 324

Kormendy, J., 1982, ApJ, 257, 75

Kormendy, J. \& Kennicutt, R. C. 2004, ARAA 42, 603

Kormendy, J. \& Illingworth, G. 1982, ApJ 256, 460

Kormendy, J. \& Gebhardt, K. 2001, in: J. C. Wheeler \& H. Martel (eds.), 20th Texas Symposium on Relativistic Astrophysics, AIP Conference Proceedings, Vol. 586., 363 